\title{
Fidelity Analysis of Additive and Multiplicative Watermarked Images in Integrated Domain
}

\author{
S.K. Jayanthi ${ }^{1}$, K. Sridevi ${ }^{2}$ \\ ${ }^{I}$ (Head and Associate Professor, Department of Computer Science, Vellalar College for women, Erode, \\ Tamilnadu, India.) \\ ${ }^{2}$ (Research Scholar, Department of Computer Science, Vellalar College for women, Erode, Tamilnadu, India.)
}

\begin{abstract}
The escalation of internet has increased the usage of multimedia contents for wide range of functions. The easy access of the digital contents paves way to manipulate, edit and duplicate the contents using available image processing software. Watermarking process ensures copy protection and data authentication to the multimedia content by embedding watermark. The present paper proposes fidelity analysis of additive and multiplicative watermarked images in the integrated domain using Peak Signal to Noise Ratio measures. This paper first embeds additive watermark in spatial domain and multiplicative watermark in frequency domain, then vice versa. The later one provides good fidelity and less image degradation by achieving acceptable Peak Signal to Noise Ratio values.
\end{abstract}

Keywords: Digital watermarking, Discrete Wavelet transform, Peak Signal to Noise Ratio;

\section{Introduction}

The success of the internet allows for the rampant distribution of multimedia data in an effortless manner. Due to the open environment of internet downloading, copyright protection introduces a new set of challenging problems regarding security and illegal distribution of privately owned images. One potential solution for declaring the ownership of the images is to use watermarks [1]. The process of watermarking involves the modification of multimedia data to embed a watermark containing key information such as authentication or copyright codes [10]. Watermarks are embedded in such a way that there is no perceptible difference between the original images and watermarked and also it is difficult to remove or alter without damaging the original image [2]. Watermarks exhibit the following properties: imperceptible, inseparable from the work in which they are embedded, and it undergoes the same transformation as the works [20].

Digital image watermarking is applied on diverse fields such as content archiving, meta data insertion, copyright protection, medical image authentication, tamper detection, broadcast monitoring, and digital fingerprinting [11]. The watermarked images are distorted by intentional or unintentional attacks like image processing attacks, geometric transformation, cryptographic attacks and protocol attacks [21]. Digital watermarking system should exhibit properties like transparency, robustness, security, capacity, invert ability, complexity and possibility of verification [3].

\section{General Watermarking System}

Fig.1 shows the general watermarking system [3], which consists of an embedder that takes host image, watermark message, key as inputs, the watermarked image $\mathrm{A}$ is attacked by noise during communication and detector which gets noisy watermarked image B as input, detects or checks for presence of watermark or extracts the watermark encoded in the image using the key. The watermark embedded can be a pseudo-random gaussian sequence, binary image or a gray scale image.

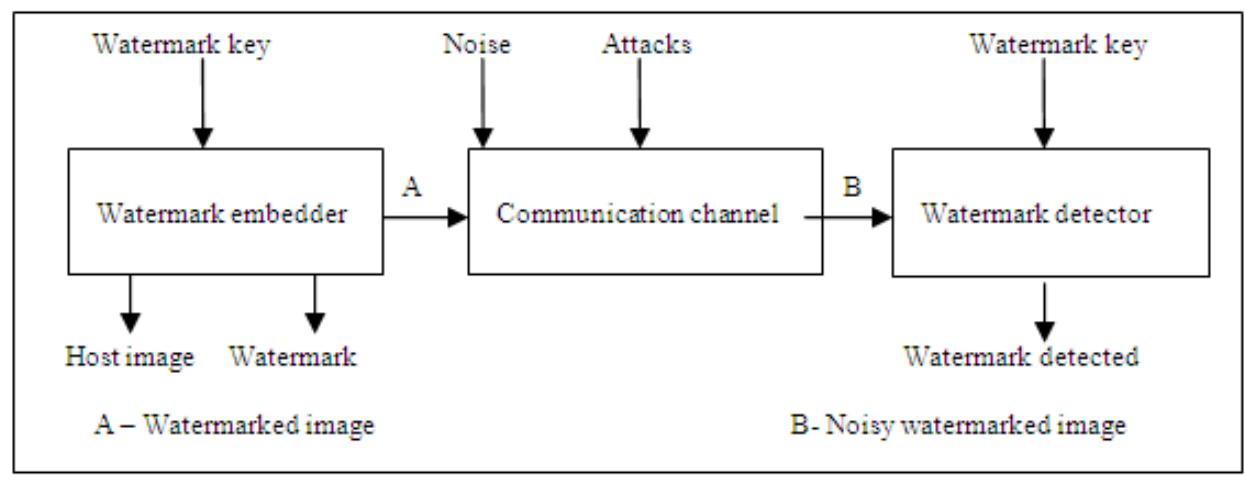

Fig .1 A generic watermarking system 


\section{Image Watermarking Domain}

Digital Image Watermarking embeds a watermark into the host image either in spatial domain or frequency domain. In spatial domain, watermark is directly embedded into the image pixels by altering the intensity values of the pixels. Least Significant Bit (LSB) substitution [12], changing paired pixels [13], [4], coding by textured blocks [14], additive approach [21], simple integer transform using reversible contrast mapping [8], dual watermarking techniques [18] are spatial domain watermarking techniques. In frequency domain watermarking, the spatial image is transformed into frequency coefficients using transforms like Discrete Fourier Transform (DFT), Discrete Cosine Transform (DCT), and Discrete Wavelet Transform (DWT) and watermark is embedded into transformed coefficients. Spread spectrum technique [17], visible watermark technique in DCT domain [19], invisible watermark technique in DWT coefficients [5], robust watermarking using wavelet transform and edge detection [6] high capacity reversible watermarking [9], are frequency domain watermarking techniques.

\section{Related Work}

Kankanhalli et.al proposed DCT domain watermarking by embedding the watermark based on texture, edge and luminance information of the image. The image is classified into $8 \times 8$ blocks and watermark is embedded depending upon the sensitivity of blocks to image distortion [19]. The algorithm uses DCT domain but edge information of the image can be predicted well using wavelet decomposition.

W.Zhu et al suggested an invisible watermarking technique. The watermark is Gaussian distributed random sequence and inserted in DWT coefficients. The watermark is added to every high pass wavelet coefficients and is visually invisible. The watermarked image is subjected to attacks like compression and halftoning, still the watermark is identified correctly [5]. The results of the algorithm against image processing attacks like cropping, scaling, dithering and wrapping are not available.

Cox et al proposed a spread spectrum technique to embed the watermark in DCT domain using multiplicative approach. The significant coefficients of the transformed image using DCT are selected and the watermark of pseudo random real numbers is embedded using the multiplicative approach with a scaling factor [17]. The watermarked image withstands scaling, clipping, compression attacks.

Ming-Shing Hsieh et al proposed hiding digital watermarks using multi-resolution wavelet transform. A multi-energy watermarking scheme based on the qualified significant wavelet tree technique is used for embedding in wavelet domain. The watermarked image is robust to signal distortions, compression, cropping and filtering attacks [1]. The approximation details of the decomposition are not considered for watermark embedding.

Frank Y.Shih et al developed combinational image watermarking in spatial and frequency domain, in which watermark is split into two parts, one is embedded in the spatial domain using LSB technique, the other watermark and the spatial watermarked image is transformed to DCT domain and divided into equal blocks. Each block of watermark is attached to the DCT domain block [16]. The watermarked image has less degradation and resists cropping attack. The algorithm is enhanced with random permutation of watermarks. The details about whether the algorithm resists against other attacks like filtering, compression and noise are not indicated.

John Ellinas proposed a robust watermarking algorithm using wavelet transforms. The algorithm embeds the watermark to the coefficients that lie on the edges, where distortions are less noticeable. The method shows results in terms of robustness and transparency to various attacks such as median filtering, Gaussian noise, JPEG compression and geometrical transformations [6].

S.K. Jayanthi et al proposed dual domain and high capacity image watermarking using additive approach. The algorithm embeds dual watermarks in spatial and frequency domains using additive approach. DWT is applied on the spatially watermarked image. The watermarking system is tested for fidelity and robustness. The algorithm embeds watermark with acceptable PSNR values and with stand attacks like cropping, compression and noise attacks [15].

\section{The Proposed System}

The proposed system embeds watermarks in integrated domains of the cover image. Fidelity analysis is performed on the watermarked images to measure image degradation. Spatial additive watermark embedding and DWT multiplicative embedding is done in first technique and the embedding methods are interchanged in second technique. The fidelity of the watermarked images is measured using Peak Signal to Noise Ratio (PSNR). Watermarking techniques using the additive and multiplicative approach on spatial and Discrete Wavelet Transform (DWT) domain are presented. 


\subsection{Discrete Wavelet Transform}

The spatially watermarked image is decomposed into DWT coefficients using daubechies wavelets. The wavelet transform decomposes the image into three spatial directions, i.e. horizontal, vertical and diagonal [22] along with approximation details. With multi-resolution analysis, image can be represented at more than one resolution level. Magnitude of DWT coefficients is larger in the lower bands (LL) at each level of decomposition and is smaller for other bands ( $\mathrm{HH}, \mathrm{LH}$, and $\mathrm{HL})$ [7].

\subsection{Additive Approach}

The cover image pixel values $h(i, j)$ is altered by adding watermark pixel values $w(i, j)$. The additive approach [21] is done as follows.

$$
h^{*}(i, j)=h(i, j)+(\alpha(i, j) * w(i, j))
$$

$\alpha$ (i) represents the scaling factor and the allowable amount of distortion while embedding in the image. $h *(i, j)$ represents the watermarked image pixels values. The value ranges from 0.001 to 0.005 are experimented for PSNR values.

\subsection{Multiplicative Approach}

The multiplicative approach [21] is performed as follows.

$$
h^{*}(i, j)=h(i, j) *(1+(\alpha(i, j) * w(i, j))
$$

$\alpha$ (i) represents the scaling factor, $h *(i, j)$ represents pixels of the watermarked image, $h(i, j)$ represents the pixels cover image, $w(i, j)$ represents the pixels of watermark image.

\subsection{Integrated domain watermarking using additive in spatial and multiplicative in frequency domain.}

Step1. Watermark image $\mathrm{W}_{1}$ is a gray scale image of size $256 \times 256$.

Step2. Watermark $\mathrm{W}_{1}$ is inserted into the spatial domain of the host image $\mathrm{H}$ of size $256 \mathrm{x} 256$ using additive approach to obtain $\mathrm{H}_{\mathrm{S}}$.

Step3. $H_{S}$ is decomposed into frequency coefficients using discrete wavelet transform to obtain $H_{D W T}$. First level decomposition contains LL1, LH1, HL1, HH1 bands.

Step4. The second watermark $\mathrm{W}_{2}$, gray scale image of size $256 \times 256$, is decomposed into discrete wavelet coefficients.WLL1 of watermark coefficients are embedded into LL1 coefficients of first level $\mathrm{H}_{\text {DWT }}$ using multiplicative approach.

Step5.The image is transformed to spatial domain using inverse DWT. $\mathrm{H}_{\mathrm{SF} 1}$ becomes the final watermarked image.

Step6. The spatial domain watermarked image $\mathrm{H}_{\mathrm{S}}$ and the watermark $\mathrm{W}_{2}$ is further decomposed into second and third level of discrete wavelet coefficients. Steps 4 and 5 are repeated to obtain $\mathrm{H}_{\mathrm{SF} 2}$ and $\mathrm{H}_{\mathrm{SF} 3}$ watermarked images.

\subsection{Integrated domain watermarking using multiplicative in spatial and additive in frequency domain} The steps followed in the technique 5.4 remains same except the following changes.

Step 2 of technique A is done using multiplicative approach. Step 4 of 5.4 is performed using additive approach.

\subsection{Extracting Technique of Integrated Domain image watermarking:}

Step1. The watermarks W2 and W1 are extracted using equations (3) and (4) for the first technique

$$
\begin{aligned}
& \mathrm{W} 2=\left(\mathrm{H}_{\mathrm{SF}} / \mathrm{Hs}\right)-1 / \text { scaling factor } \\
& \mathrm{W} 1=(\mathrm{Hs}-\mathrm{H}) / \mathrm{scaling} \text { factor }
\end{aligned}
$$

W1, W2 - Watermark one and Watermark two

$$
\begin{array}{ll}
\mathrm{H}_{\mathrm{SF}} & \text { - Dual Domain Watermarked image } \\
\mathrm{Hs} & \text { - Spatial Domain Watermarked image } \\
\mathrm{H} & \text { - Original image }
\end{array}
$$

Correlation Coefficient (CC) between the extracted and original watermark is calculated using equation (5)

$$
C C=\frac{\sum_{i} \sum_{j} W(i, j) * E W(i, j)}{\sqrt{\sum_{i} \sum_{j} W(i, j)^{2} * \sum_{i} \sum_{j} E W(i, j)^{2}}}
$$

$\mathrm{W}$ - Original watermark

EW- Extracted watermark 
Step2. The watermarks of second technique are extracted from the above equations by applying the equation (4) to extract W2 and equation (3) to extract the W1.

\section{Results and Discussions}

Fig. 2 to Fig. 8 shows the integrated domain watermarking using spatial additive and frequency multiplicative approach. The two watermarks are extracted using equations (3) and (4) with correlation measure equal to one.

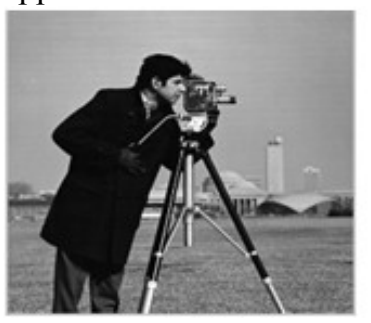

Fig.2. Original image

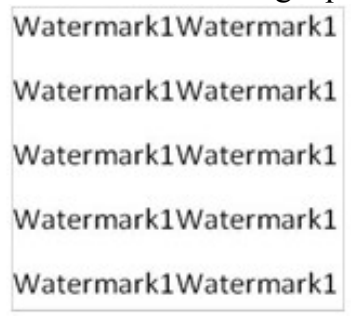

Fig.3. Watermark 1

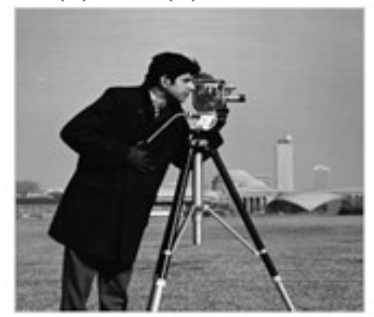

Fig.4. Spatially watermarked
Watermark2Watermark2

Watermark2Watermark2

Watermark2Watermark2

Watermark2Watermark2

Watermark2Watermark2

Fig.5. Watermark 2 image using additive approach

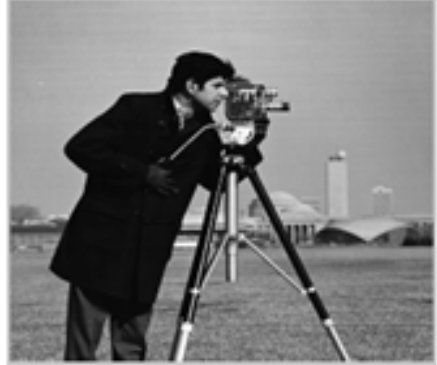

Fig.6.Integrated watermarked image using multiplicative appraoch

Watermark2Watermark2
Watermark2Watermark2
Watermark2Watermark2
Watermark2Watermark2
Watermark2Watermark2

Fig.7.Extractedwatermark2

\section{Watermark1Watermark1 \\ Watermark1Watermark1 \\ Watermark1Watermark1 \\ Watermark1Watermark1 \\ Watermark1Watermark1}

Fig.8. Extracted watermark 1

Fig.9 to Fig. 15 shows the integrated domain watermarked image using spatial multiplicative and frequency additive approach. The watermarks are extracted using equations (2) and (1) with correlation coefficient equal to one.

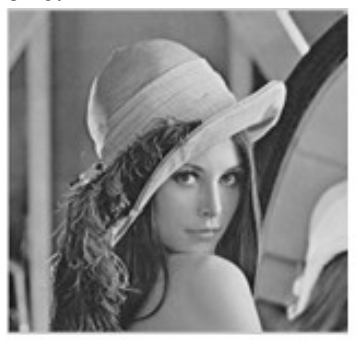

Fig.9. Original image

Watermark1Watermark1
Watermark1Watermark1
Watermark1Watermark1
Watermark1Watermark1
Watermark1Watermark1

Fig.10. Watermark 1

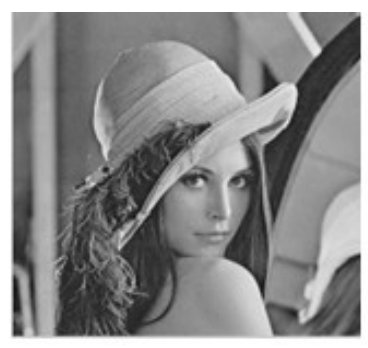

Fig.11. Spatially watermarked
Watermark2Watermark2 Watermark2Watermark2 Watermark2Watermark2 Watermark2Watermark2 Watermark2Watermark2

Fig.12. Watermark 2 image using multiplicative approach

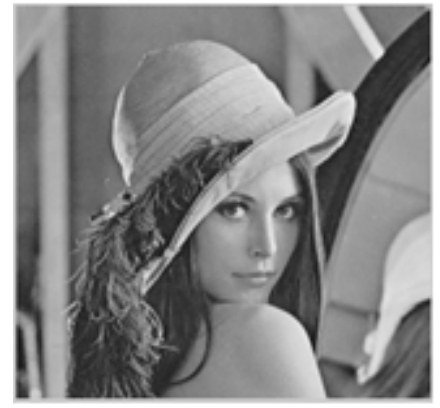

Fig.13. Integrated watermarked image using additive approach

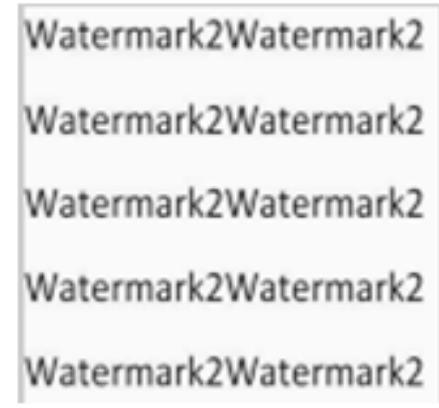

Fig.14. Extracted watermark 2

\section{Watermark1Watermark1 \\ Watermark1Watermark1 \\ Watermark1Watermark1 \\ Watermark1Watermark1 \\ Watermark1Watermark1}

Fig.15. Extracted watermark1 


\section{Performance Analysis}

Error measures such as Peak Signal to Noise Ratio (PSNR) is used to compute the image distortion in the watermarked images. PSNR values are calculated for additive and multiplicative applied spatial domain, DWT domain and integrated domain watermarked images. Values from Table1 to Table4 illustrate the performance of the proposed integrated domain image watermarking by varying $\alpha$ values from 0.001 to 0.005 . PSNR is computed as follows:

$$
P S N R=10 \log _{10}\left[\frac{\sum_{i=1}^{N} \sum_{j=1}^{N}[h *(i, j)]^{2}}{\sum_{i=1}^{N} \sum_{j=1}^{N}\left[h(i, j)-h^{*}(i, j)\right]^{2[]}}\right]
$$

$h *(i, j)$ - pixel value of watermarked image

$h(i, j)$ - pixel value of original image

$\mathrm{N} \times \mathrm{N}$ - size of the image

Recommended PSNR values must be $>35$ Decibel

Table 1. Psnr Values Of Spatial Domain Additive And Multiplicative Approach

\begin{tabular}{|c|c|c|}
\hline $\begin{array}{c}\alpha \\
\text { falues (scaling } \\
\text { factor) }\end{array}$ & PSNR values of spatial additive approach & $\begin{array}{c}\text { PSNR values of } \\
\text { spatial multiplicative approach }\end{array}$ \\
\hline 0.001 & 54.9106 & 60.4994 \\
\hline 0.002 & 48.9033 & 54.4867 \\
\hline 0.003 & 45.3949 & 50.9728 \\
\hline 0.004 & 42.9094 & 48.4820 \\
\hline 0.005 & 40.9846 & 46.5517 \\
\hline
\end{tabular}

The PSNR values of spatial multiplicative approach are higher and acceptable values when compared to the spatial additive approach. The values reveal that spatial multiplicative approach embedding produces less image degradation.

Table 2. Psnr Values Of Dwt Domain Additive And Multiplicative Approach

\begin{tabular}{|c|c|c|}
\hline $\begin{array}{c}\text { a values (scaling } \\
\text { factor) }\end{array}$ & $\begin{array}{c}\text { PSNR values of DWT additive } \\
\text { approach }\end{array}$ & $\begin{array}{c}\text { PSNR values of } \\
\text { DWT multiplicative approach }\end{array}$ \\
\hline 0.001 & 55.0335 & 54.6525 \\
\hline 0.002 & 49.0261 & 48.6475 \\
\hline 0.003 & 45.5175 & 45.1412 \\
\hline 0.004 & 43.0320 & 42.6580 \\
\hline 0.005 & 41.1070 & 40.7352 \\
\hline
\end{tabular}

The PSNR values of frequency domain watermarked image using additive and multiplicative approach shows acceptable values and less image degradation.

Table 3. Psnr Values Of Integrated Spatial Additive And Frequency Multiplicative Approach

\begin{tabular}{|c|c|c|c|}
\hline \multirow{2}{*}{$\begin{array}{c}\boldsymbol{\alpha} \text { values } \\
\text { (scaling factor) }\end{array}$} & I level & II level & III level \\
\cline { 2 - 4 } & 49.0999 & 45.5805 & 42.9429 \\
\hline 0.001 & 43.1002 & 39.5936 & 36.9813 \\
\hline 0.002 & 39.5993 & 36.1054 & 33.5181 \\
\hline 0.003 & 37.1214 & 33.6401 & 31.0775 \\
\hline 0.004 & 35.2040 & 31.7352 & 29.1972 \\
\hline
\end{tabular}

The spatially watermarked image is decomposed into first, second and third level DWT coefficients. Multiplicative approach is used to embed the watermarks in the DWT coefficients. The PSNR values of the watermarked images tend to decrease on further decomposition to II and III levels. The reason for decrease in values is that the image coefficients get doubled when multiplied with the watermark coefficients, which leads to high image degradation on further levels of watermark embedding. 
Table 4. Psnr Values Of Integrated Spatial Multiplicative And Frequency Additive Approach

\begin{tabular}{|c|c|c|c|}
\hline \multirow{2}{*}{$\begin{array}{c}\text { a values } \\
\text { (scaling } \\
\text { factor) }\end{array}$} & I level & II level & III level \\
\cline { 2 - 4 } & 51.6429 & 51.6847 & 51.7102 \\
\hline 0.001 & 45.6434 & 45.6853 & 45.7108 \\
\hline 0.002 & 42.1427 & 42.1846 & 42.2101 \\
\hline 0.003 & 39.6650 & 39.7068 & 39.7324 \\
\hline 0.004 & 37.7479 & 37.7897 & 37.8152 \\
\hline 0.005 & &
\end{tabular}

The additive approach when used to embed watermark in DWT coefficients tends to produce acceptable PSNR values. On comparison of PSNR values of Table 3 and 4, it reveals that spatial multiplicative and DWT additive approach produces high and suggested PSNR measures since distortion to the DWT coefficients are less by the usage of additive approach.

\section{Conclusion}

The proposed work analyzes fidelity property of two integrated domain watermarking techniques. Spatial watermark embedding using multiplicative approach with DWT coefficients watermark embedding using additive approach produces acceptable PSNR measures and less image degradation compared to the other technique. The alpha values are varied from 0.001 to 0.005 to test the imperceptibility of the integrated domain watermarked images. The watermark embedded is extracted with correlation measure equal to one.

\section{Journal Papers:}

\section{References}

[1] Ming-Shing Hsieh, Din-Chang Tseng et al, Hiding Digital Watermarks using Multiresolution Wavelet Transform, IEEE Transactions on Industrial Electronics, Vol.48,No.5, October 2001.

[2] Christine Podichuk, Edward J.Delp, Digital Watermarking Algorithms and Applications, IEEE Signal Processing Magazine, 1053.5888/01 2001 .

[3] Prabhishek Singh, R.S Chadha, A Survey of Digital Watermarking Techniques, Applications and Attacks, International Journal of Engineering and Innovative Technology, 2277-3754, Vol.2, Issue 9 March 2013

[4] W. Bender et al, Techniques for data hiding, IBM Systems Journal. Vol.35 1996.

[5] W.Zhu,et al, Multiresolution Watermarking for Images and Videos: A Unified Approach, IEEE Tran. on Circuits \& Systems for Video Technology.Vol 9,No.4, June 1999.

[6] John.N.Ellinas, A Robust Wavelet-Based Watermarking Algorithm using Edge Detection, World Academy of Science, Engineering and Technolgy 34, 2000.

[7] Vaishali.S.Jabade.et al, Literature Review of Wavelet Based Digital Image Watermarking Techniques, International Journal of Computer Applications (0975-8886)) Vol.31-Oct 2011.

[8] Dinu Coltuc and Jean-Marc Chassery, Very Fast Watermarking by Reversible Contrast Mapping, IEEE Signal Processing Letters, Vol. 14,NO.4, April 2007

[9] Sunil Lee Chang D.Yoo, Ton Kalker, Reversible Image Watermarking by Based on Integer-to -Integer Wavelet Transform, Information Forensics and Security, IEEE Transactions on. Vol. 2, Issue: 3, Sept 2007.

\section{Conference Proceedings:}

[10] Deepa Kundur, Dimitrios Hatzinakas, Digital Watermarking using Multiresolution Wavelet Decomposition, Proceedings of IEEE International conference on Vol. 5 may 1998.

[11] Vidyasagar M. Potadar et al, A Survey of Digital Image Watermarking Techniques, $3^{\text {rd }}$ International Conference on Industrial Informatics, IEEE 2005

[12] Wolfgang. R and E. Delp, A Watermarking Technique for Digital Imagery: Further Studies, In Proc.Int.Conf. Imaging Science, Systems and Technology. Los Vegas. NV 1997.

[13] Pitas. I and T.Kaskalis, Applying Signatures on Digital images, In Proc. IEEE Workshop Nonlinear Signal and Image Processing 1995.

[14] Caronni.G, Assuring Ownership Rights for Digital Images, In Proc.Reliable IT Systems, Germany: Viewveg, 1995.

[15] S.K. Jayanthi, Sridevi.K ,Dual Domain and High Capacity Image Watermarking using Additive Approach, Proceedings 2014 International Conference on Information Science, Electronics and Electrical Engineering. April 26-28 2014.

[16] Shih.F.Y. K.Y.Yu and Y.Z.Chen, Combinational Image Watermarking in the Spatial and Frequency Domain, Pattern Recognition 362003:969.

[17] Cox et al, A Secure Robust Watermark for Multimedia, In Proc. First Int. Workshop Information Hiding, Cambridge, UK 1996,185 .

[18] S.P Mohanty, et al., A Dual Watermarking Technique for Images , Proc. $7^{\text {th }}$ ACM International Multimedia Conference, ACMMM'99, Part 2, pp.49-51, Orlando, USA, Oct. 1999.

[19] M.Kankanhali, et al., "Adaptive Visible Watermarking of Images “, Proc. Of IEEE Int. Conf. On Multimedia Computing Systems. ICMCS-99, Cento Affari, Florence, Italy. June 1999.

Books:

[20] Ingemar J.Cox, Mathew L.Miller, Jeffrey A. Bloom, Digital Watermarking, Morgan Kaufman Publishers, 2002.

[21] Frank Y.Shih, Digital watermarking and Stegnography - Fundamentals and Techniques, CRC Press, First Indian Reprint 2012.

[22] Rafel.C.Gonzalez, Richards.E.Woods, Digital Image Processing, Pearson Education, $3^{\text {rd }}$ Edition 2008. 